\title{
ANÁLISE DA FUNÇÃO LOG-LOGÍSTICA PARA MODELAGEM DA DISTRIBUIÇÃO DE DIÂMETROS ${ }^{1}$
}

\begin{abstract}
Daniel Henrique Breda Binoti ${ }^{2}$, Mayra Luiza Marques da Silva Binoti ${ }^{3}$ e Helio Garcia Leite ${ }^{4}$
RESUMO - Objetivou-se neste estudo avaliar a eficiência da função log-logística com dois e três parâmetros, $\gamma=$ dapmin e truncada à direita para a descrição da estrutura diamétrica de povoamentos equiâneos, bem como ajustar um modelo de distribuição diamétrica utilizando as funções. A modelagem realizada pela função loglogística foi comparada com a modelagem realizada com a função Weibull. Para isso, utilizaram-se dados de parcelas permanentes de clones de Eucalyptus, mensuradas em seis ocasiões. A aderência das funções aos dados foi comprovada pela aplicação do teste de Kolmogorov-Smirnov (K-S). Os valores médios da estatística do teste K-S foram 0,$0901 ; 0,0997 ; 0,1297$; e 0,0616 para a função com 2P, 3P, $a=$ dapmin e truncada à direita, respectivamente. Para a função Weibull, obteve-se a média de 0,0638 para a estatística do teste K-S. A função log-logística de dois parâmetros pode ser utilizada na modelagem da estrutura diamétrica de povoamentos de eucalipto.
\end{abstract}

Palavras-chave: Weibull; Truncamento; Estrutura diamétrica.

\section{USE OF LOG-LOGISTIC FUNCTION FOR MODELING DIAMETER DISTRIBUTION}

\begin{abstract}
The objective of this study was to evaluate the efficiency of the log-logistic function with 2 and 3 parameters, $\gamma=$ dapmin right truncated to describe the diameter structure of even-aged stands and set a diametric distribution model using the functions. The modeling performed by the log-logistic function was compared to the modeling performed with the Weibull function. For this, we used data from permanent plots of Eucalyptus clones, measured on six occasions. The grip of the functions to the data was confirmed by applying the Kolmogorov-Smirnov $(K-S)$ test. The average values of the $K-S$ test statistic was 0.0901, 0.0997, 0.1297, and 0.0616 for the function with $2 P, 3 P, \tilde{a}=$ dapmin right truncated, respectively. For the Weibull function an average of 0.0638 was obtained for the $K$ $S$ test statistic. The log-logistic function of two parameters can be used to simulate the diametric structure of eucalyptus plantations.
\end{abstract}

Keywords: Weibull; Truncation; Structure diameter.

\footnotetext{
${ }^{1}$ Recebido em 07.04.2013 aceito para publicação em 07.05.2015.

${ }^{2}$ DAP Engenharia Florestal, Viçosa, MG - Brasil. E-mail: <danielhbbinoti@gmail.com>.

${ }^{3}$ Universidade Federal do Espírito Santo, Centro de Ciências Agrárias (CCA), Departamento de Ciências Florestais e da Madeira, Vitória, ES - Brasil.E-mail: <mayrabinoti@gmail.com>.

${ }^{4}$ Universidade Federal de Viçosa, Centro de Ciências Agrárias, Departamento de Engenharia Florestal,Viçosa, MG - Brasil. E-mail: <Hgleite@gmail.com>.
} 


\section{INTRODUÇÃO}

Modelos de crescimento e produção florestal são usados para a predição do crescimento do povoamento em função de características do local, do povoamento e dos tratos silviculturais e constituem importantes ferramentas para a tomada de decisão no manejo florestal sustentável. Esses modelos podem ser classificados em três tipos: modelos em nível de povoamento, modelos de distribuição de diâmetros e modelos de árvores individuais (DAVIS et al., 2001; CAMPOS; LEITE, 2009).

A maioria dos modelos publicados e empregados no Brasil é do tipo povoamento total. Isso se justifica pelo objetivo do manejo, que na maioria das vezes requer projeções de volume para um ou dois usos da madeira, sempre em nível de povoamento. Apesar disso, algumas vezes as estimativas oriundas desses modelos são insuficientes para avaliações econômicas detalhadas, visto que alguns coeficientes operacionais das operações de colheita e transporte são influenciados pelas dimensões das árvores (DAVIS et al., 2001; BETTINGER et al., 2009).

Quando o manejo requer informações detalhadas sobre a estrutura e volume do povoamento, bem como a quantificação de multiprodutos, é mais indicado o emprego de modelos de distribuição de diâmetros (CAMPOS; LEITE, 2009).

A construção de um modelo de distribuição de diâmetros requer o emprego de distribuições estatísticas. Entre diversas funções densidade de probabilidade $(f d p)$, utilizadas para a descrição da estrutura diamétrica de povoamentos equiâneos, destacam-se: Weibull, normal, log-normal, gama, SB Johnson's, beta, loglogística, Cauchy, Frechet, Erlang, Rayleigh e hiperbólica (CLUTTER;ALLISON, 1974; HAFLEY; SCHREUDER, 1977; CAMPOS; TURNBULL, 1981; MATNEY; SULLIVAN, 1982; CAO, 2004; NOGUEIRA et al., 2005; PALAHÍ et al., 2006, 2007; BINOTI 2008; LEITE et al., 2010).

A função densidade de probabilidade log-logística em sua forma completa (3P) pode ser escrita da seguinte forma (SHOUKRI et al., 1988; JOHNSON et al., 1995; BINOTI, 2008):

$$
f(x)-\frac{\alpha}{\beta}\left(\frac{x-\gamma}{\beta}\right)^{\alpha-1}\left(1+\left(\frac{x-\gamma}{\beta}\right)^{\alpha}\right)^{-2}
$$

em que $\alpha$ é o parâmetro de forma $(\alpha>0)$, â é o parâmetro de escala $(\beta>0)$ e $\gamma$ é o parâmetro de locação. A supressão do parâmetro de locação resulta na função log-logística de dois parâmetros $(2 \mathrm{P})$ e pode ser expressa da seguinte forma:

$$
f(x)=\frac{\alpha}{\beta}\left(\frac{x}{\beta}\right)^{\alpha-1}\left(1+\left(\frac{x}{\beta}\right)^{\alpha}\right)^{-2}
$$

O parâmetro de locação pode também ser substituído pelo diâmetro mínimo da parcela (dapmin), resultando na função log-logística com $\gamma=$ dapmin (BINOTI, 2008):

$$
f(x)=\frac{\alpha}{\beta}\left(\frac{x-d a p \min }{\beta}\right)^{\alpha-1}\left(1+\left(\frac{x-d a p \min }{\beta}\right)^{\alpha}\right)^{-2}
$$

O truncamento da função pelo diâmetro máximo (dapmax) da parcela pode ser definido da seguinte forma (BINOTI, 2008):

$$
f(x)=\frac{\alpha\left(\begin{array}{c}
x-\gamma \\
\beta
\end{array}\right)^{\alpha-1}\left(1+\left(\begin{array}{c}
x-\gamma \\
\beta
\end{array}\right)^{\alpha}\right)^{-2}}{\left(1+\left(\frac{\beta}{d a p \max -\gamma}\right)^{\alpha}\right)^{-1}}
$$

A função acumulativa da função log-logística 3P pode ser expressa na equação 5 (JOHNSON et al., 1995):

$$
F(x) 1=\left(+\left(\frac{\beta}{x-\gamma}\right)^{\alpha}\right)^{-1}
$$

Objetivou-se neste estudo avaliar a eficiência da função log-logística completa, log-logística de dois parâmetros e log-logística tendo como parâmetro de locação o diâmetro mínimo e a log-logística truncada, para a descrição da estrutura diamétrica de povoamentos equiâneos de Eucalyptus, bem como propor um modelo de distribuição diamétrica utilizando as funções.

\section{MATERIAL E MÉTODOS}

Utilizaram-se neste estudo dados obtidos de 400 parcelas retangulares permanentes de $340 \mathrm{~m}^{2}$, instaladas em povoamentos de híbridos de Eucalyptus grandis $\mathrm{x}$ Eucalyptus urophylla, na região Centro-Oeste do Estado de Minas Gerais, Brasil. O relevo varia de forte- 
ondulado a montanhoso. O solo predominante é o Cambissolo, com precipitação média anual de $1.280 \mathrm{~mm}$, temperatura média anual de $20,3^{\circ} \mathrm{C}$, umidade relativa média anual de $71,2 \%$. Os povoamentos foram estabelecidos no arranjo espacial de $3 \times 3 \mathrm{~m}$, com rotação de corte média de 7 anos. Nessa idade, a produtividade média nesses povoamentos varia entre $25 \mathrm{e} 50 \mathrm{~m}^{3} \cdot \mathrm{ha}^{-1}$. ano $\mathrm{H}^{-1}$. As medições de diâmetros foram efetuadas nas árvores com diâmetro a 1,3 de altura (dap) acima de $5 \mathrm{~cm}$, nas idades médias de $28,40,52,64,76$ e 84 meses.

Os dados de dap observados em cada parcela foram agrupados em classe com amplitude de $1,0 \mathrm{~cm}$. Ajustouse a função log-logística nas formas: log-logística 3P, log-logística $2 \mathrm{P}, \log$-logística $\gamma=$ dapmin e log-logística truncada à direita. Ajustou-se também um modelo de distribuição de diâmetros utilizando a função Weibull, para fins comparativos, a qual pode ser descrita como:

$$
f(x)=\frac{\alpha}{\beta}\left(\frac{x}{\beta}\right)^{\alpha-1} e^{\left(-\left(\frac{x}{\beta}\right)^{\alpha}\right)}
$$

em que $\beta$ é o parâmetro de escala $(\beta>0)$ e $a ́$, o parâmetro de forma $(\alpha>0)$.

Os ajustes foram feitos pelo método da máxima verossimilhança (BINOTI, 2008; CAMPOS; LEITE, 2009; LEITE et al., 2010; BINOTI et al., 2012). Para avaliar a aderência da função aos dados, utilizou-se o teste de Kolmogorov-Smirnov (K-S) (SOKAL; ROHLF, 2012). Realizou-se a análise gráfica entre valores estimados e observados para todos os ajustes obtidos com a função log-logística e Weibull.

A construção do modelo de distribuição diamétrica baseou-se na alternativa usual de correlação dos parâmetros da $f d p$ com características do povoamento. Conforme Nogueira et al. (2005), utilizaram-se como variável dependente os parâmetros da função log-logística em uma idade futura e como variável independente, os parâmetros da função e características do povoamento em uma idade atual.

A redistribuição teórica dos diâmetros foi avaliada pela capacidade do sistema de equações em projetar o diâmetro máximo, o número total de árvores e os parâmetros da função log-logística e Weibull $(\alpha, \beta$ $e \gamma$ para qualquer idade. Construiu-se um modelo para cada configuração testada da função (log-logística $3 \mathrm{P}, \log$-logística $2 \mathrm{P}, \log$-logística $\tilde{a}=$ dapmin e loglogística truncada à direita). O coeficiente de correlação entre os valores observados e estimados e a análise gráfica dos resíduos foram utilizados para avaliar a exatidão das estimativas do sistema de equações. Para avaliar a exatidão das estimativas geradas pelas equações que compõem o modelo de distribuição de diâmetros, estimou-se também a estatística RMSE (\%) (SIIPILEHTO, 2000). O estimador utilizado foi:

$$
\operatorname{RMSE}(\%)=100 / \bar{Y} \sqrt{\frac{\sum_{i=1}^{n}\left(Y_{i}-\bar{Y}_{i}\right)}{n}}
$$

em que $\bar{Y}$ é a média da saída observada e $n$, o número total de dados.

\section{RESULTADOS}

Ajustaram-se as funções log-logística completa, log-logística de dois parâmetros e log-logística tendo como parâmetro de locação o diâmetro mínimo, a loglogística truncada e Weibull para todas as observações, sendo cada estimação comparada com a distribuição observada. Todos os ajustes resultaram em aderência aos dados pelo teste de Kolmogorov-Smirnov $(\mathrm{P}>0,01)$. Os valores médios da estatística do teste foram 0,0901 ; 0,0997; 0,1297; e 0,0616, para a função com 2P, 3P, $\gamma=$ dapmin e truncada à direita, respectivamente. Para a função Weibull, obteve-se a média de 0,0638 para a estatística do teste K-S. Três parcelas foram escolhidas aleatoriamente, cujos ajustes são apresentados na Figura 1, para as idades de medição aos 28, 40 e 52 meses.

Os modelos de distribuição diamétrica ajustados com os respectivos coeficientes de correlação e RMSE (\%) são apresentados a seguir, com exceção dos modelos da função truncada, visto que esta não apresentou correlação significativa $(p>0,05)$ entre seus parâmetros e características do povoamento.

Log-logística 3P

$$
\begin{aligned}
& \hat{\alpha}_{2}=\alpha_{1} e^{\left(-0,0001\left(I_{2}^{1,7779}-I_{1}^{1,7979}\right)\right)} R_{\hat{y y}}=0,7240 \operatorname{RSME}(\%)=27,641 \\
& \hat{\beta}_{2}=\beta_{1} e^{\left(-19,4181\left(I_{2}^{-1,0181}-I_{1}^{-1,0181}\right)\right)} \quad R_{y y}=0,7724 \operatorname{RSME}(\%)-16,176 \\
& \hat{\gamma}_{2}=\gamma_{1} e^{\left(-0,0009\left(I_{2}^{1,4546}-I_{1}^{1,4546}\right)\right)} R_{\hat{y y}}=0,8224 \quad \operatorname{RSME}(\%)=48,894
\end{aligned}
$$

Log-logística $2 \mathrm{P}$

$\hat{\alpha}_{2}=\alpha_{1} e^{\left(-0,0001\left(I_{2}^{1,7649}-I_{1}^{1,7649}\right)\right)} \quad R_{\hat{y}}=0,9703 \quad \operatorname{RSME}(\%)-11,713$

Revista Árvore, Viçosa-MG, v.39, n.5, p.943-951, 2015 


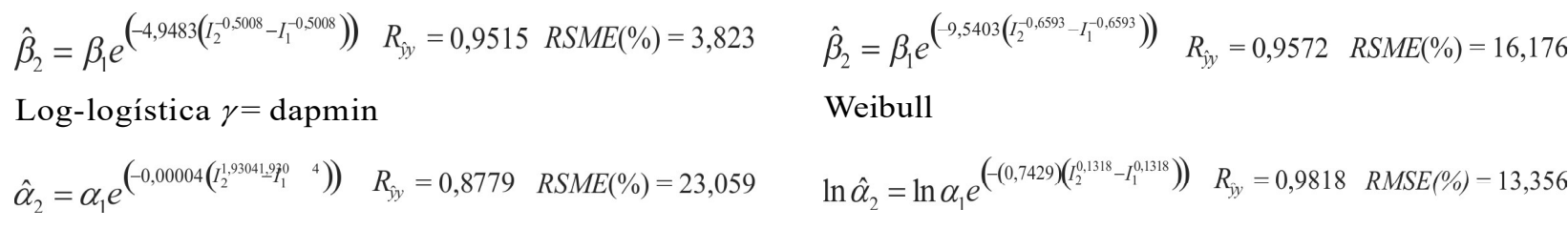

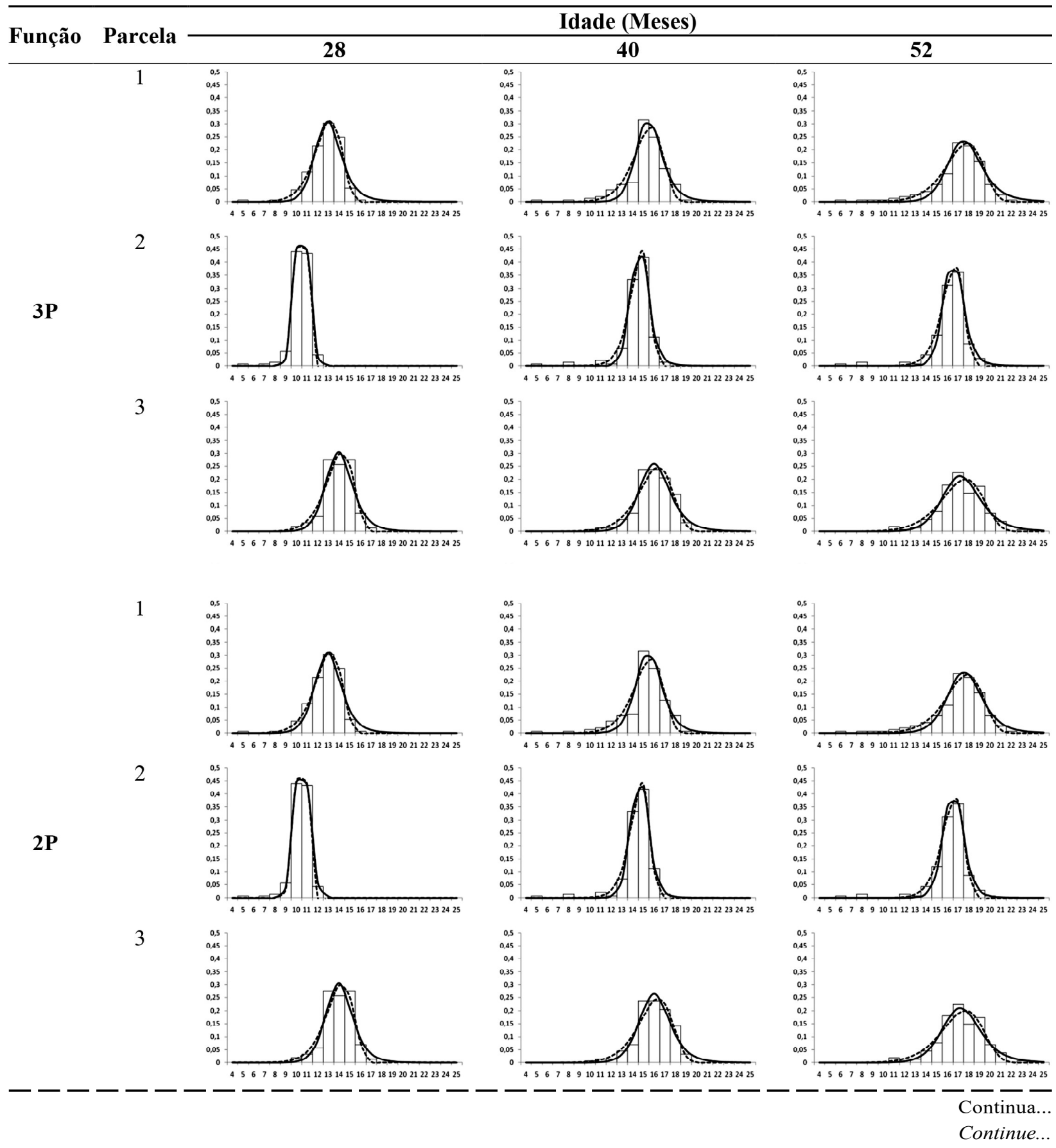

Revista Árvore, Viçosa-MG, v.39, n.5, p.943-951, 2015 
Figura 1 - Cont.

Figure 1 - Cont.

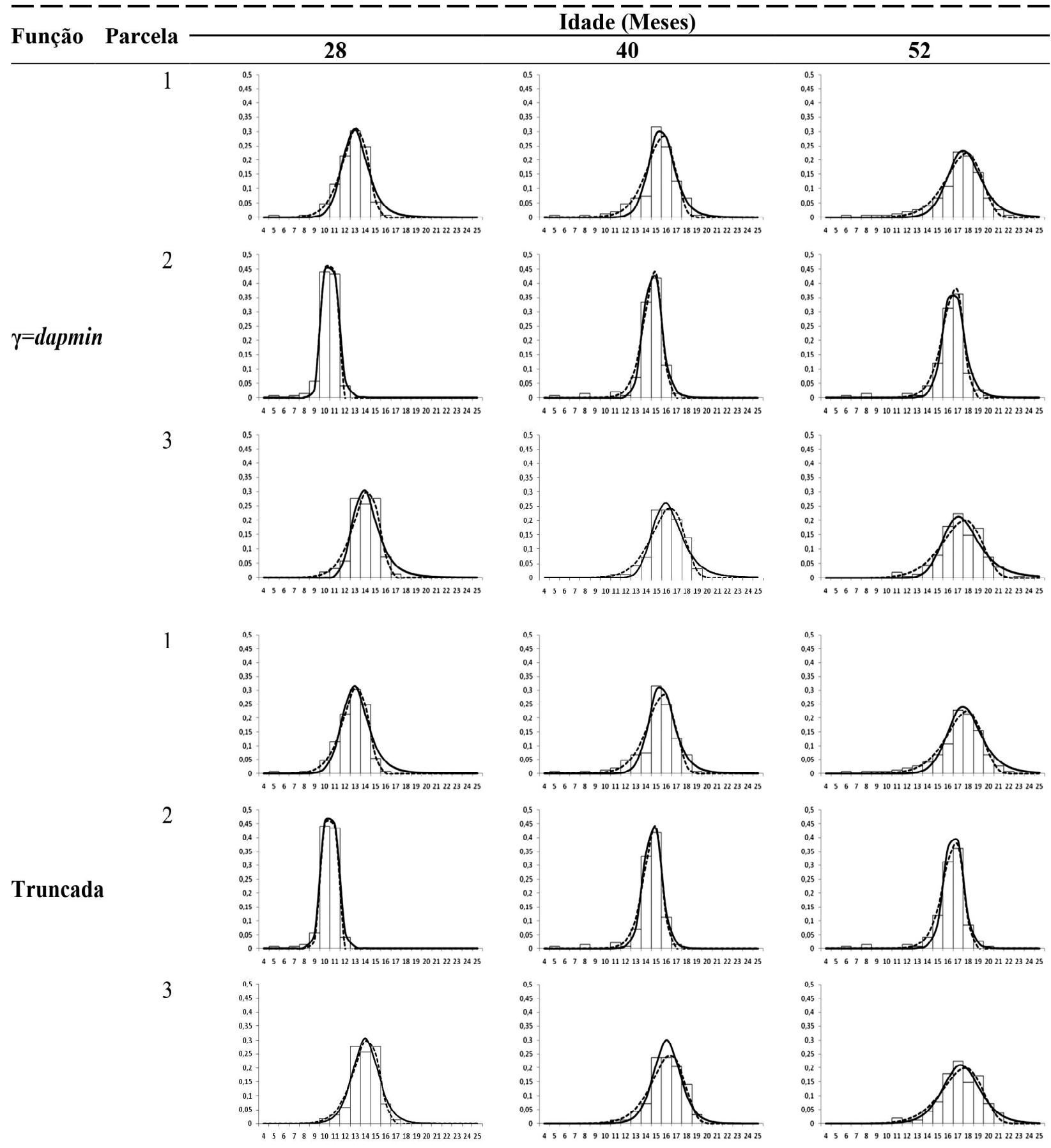

Figura 1 - Frequência observada (barras) e estimada pela função log-logística (-) e Weibull (--) para povoamentos equiâneos de eucalipto em diferentes idades.

Figure 1 - Frequency observed (bars) and estimated by log-logistic function (-) and Weibull (--) to even-aged stands of eucalypt at different ages. 
$\hat{\beta}_{2}=\beta_{1}\left(\frac{I_{1}}{I_{2}}\right)+20,70921\left(-\frac{I_{1}}{I_{2}}\right) R_{\hat{y} y}=0,9551 \quad \operatorname{RMSE}(\%)-3,829$

Características do Povoamento:

$\hat{d} \max _{2}=d \max _{1} e^{\left(-4,7152\left(I_{2}^{-0.2158}-I_{1}^{-0.2158}\right)\right)} R_{\hat{y y}}=0,9743 \quad R S M E(\%)=3,788$

$\hat{d m i n} \min _{2}=d \min _{1} \mathrm{e}^{\left(-3,3042\left(I_{2}^{0.6399-}-I_{1}^{0.06399}\right)\right)} \quad R_{\hat{j y}}=0,9517 \operatorname{RSME}(\%)=6,395$

em que $I_{1}$ e $I_{2}$ são as idades atual e futura, respectivamente, em meses; $\alpha_{1}$ e $\alpha_{2}$ são os parâmetros de forma das funções log-logística e Weibull nas idades atual e futura; $\beta_{1}$ e $\beta_{2}$ são os parâmetros de escala das funções log-logística e Weibull nas idades atual e futura; $\gamma_{1}$ e $\gamma_{2}$ são os parâmetros de locação da função log-logística nas idades atual e futura; $\operatorname{dmax}_{1}$ e $\operatorname{dmax}_{2}$ são os diâmetros máximos nas idades atual e futura, em cm, $d \min _{1}$ e $d \min _{2}$, são os diâmetros mínimos nas idades atual e futura, em cm; e ln é o logaritmo neperiano. A dispersão gráfica dos resíduos percentuais e os histogramas da dispersão dos erros de cada equação que compõe o sistema são apresentados na Figura 2. Não houve necessidade de projeção da densidade do povoamento em função do baixo índice de mortalidade observado.

\section{DISCUSSÃO}

Desde sua introdução na área florestal em 1973, a função Weibull tem sido empregada com frequência para a modelagem da distribuição de diâmetros de povoamentos (BAILEY; DELL, 1973). As justificativas para o uso convencional dessa função são a flexibilidade e a correlação significativa normalmente encontrada entre seus parâmetros e características dos povoamentos (CLUTTER; ALLISON, 1974; HAFLEY; SCHREUDER, 1977; CAMPOS; TURNBULL, 1981; MATNEY; SULLIVAN, 1982; CAO, 2004; NOGUEIRA et al., 2005; PALAHÍ et al., 2006, 2007; CAMPOS; LEITE, 2009).

A utilização do diâmetro mínimo da parcela como parâmetro de locação para o ajuste da função loglogística apresentou diminuição da qualidade dos ajustes da função, como pode ser observado pelo aumento na média da estatística do teste K-S. A comparação entre valores observados e estimados, bem como valores estimados pela função Weibull, são apresentados na Figura 1, demonstrando não haver variabilidade significativa.

Revista Árvore, Viçosa-MG, v.39, n.5, p.943-951, 2015
As parcelas selecionadas condizem com uma situação típica dos povoamentos equiâneas, com a distribuição tendendo a normal. A dinâmica de evolução tende a tornar a distribuição assimétrica à direita, em razão de as árvores de maiores dimensões apresentarem maiores taxas de crescimento radial em relação às árvores menores (GUIMARÃES, 1994). A Figura 1 demonstra que ambas as funções conseguem descrever a dinâmica do povoamento em diversas idades.

A projeção dos parâmetros da função log-logística de $2 \mathrm{P}$ apresentou desempenho superior ao modelo de distribuição de diâmetros proposto para a função Weibull, o que é comprovado pelos menores RSME (\%) obtidos pelas equações e pela análise gráfica dos resíduos (Figura 2). Esse fato garante a sustentabilidade para a recuperação dos parâmetros da função em uma idade futura, possibilitando a utilização da função para a projeção de distribuição de diâmetros em povoamentos equiâneos.

Pela análise de resíduos na Figura 2, feita para detectar tendências nos valores residuais em relação à idade, pode-se comprovar que somente as projeções dos parâmetros da função Weibull e log-logística 2P resultaram em estimativas precisas e não tendenciosas em qualquer idade. A maioria dos erros oscilou em aproximadamente $\pm 20 \%$.

O modelo proposto para a função log-logística de $3 \mathrm{P}$ apresenta tendenciosidade para a projeção do parâmetro de locação (Figura 2), inviabilizando a utilização dessa equação, e relações como outras características do povoamento ou métodos de projeção devem ser estudadas. A eficiência da função log-logística deve ser verificada para a descrição da distribuição diamétrica de povoamentos manejados sobre regime de desbaste, o que pode viabilizar a utilização da função com três parâmetros.

\section{CONCLUSÃO}

As funções log-logística 3P, log-logística de $2 \mathrm{P}$ e log-logística, tendo como parâmetro de locação o diâmetro mínimo do povoamento, podem ser utilizadas com eficiência para a descrição da distribuição de diâmetros de povoamentos de Eucalyptus, bem como a construção de modelos de distribuição de diâmetros. 


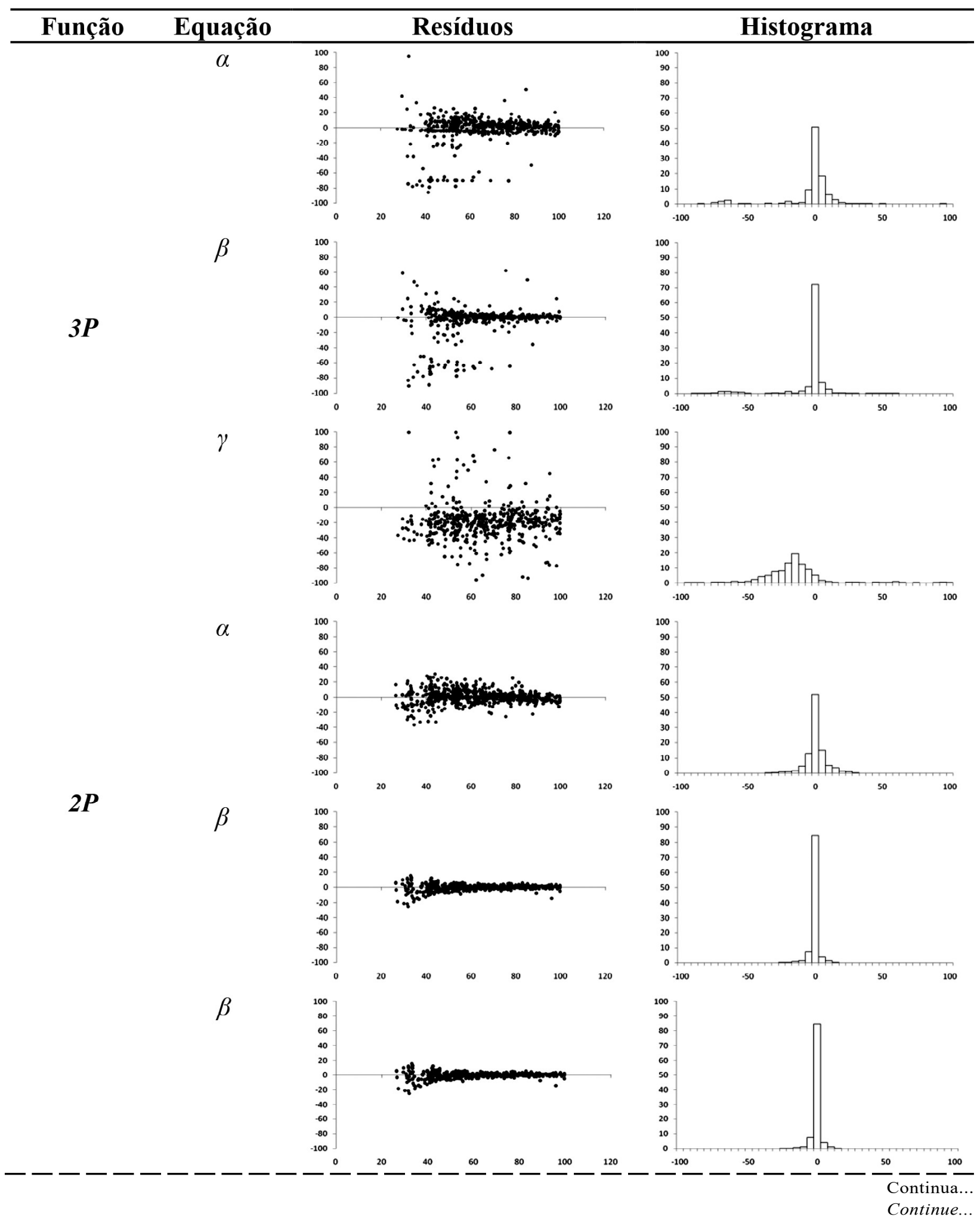


Figura 2 - Cont.

Figure 2-Cont.

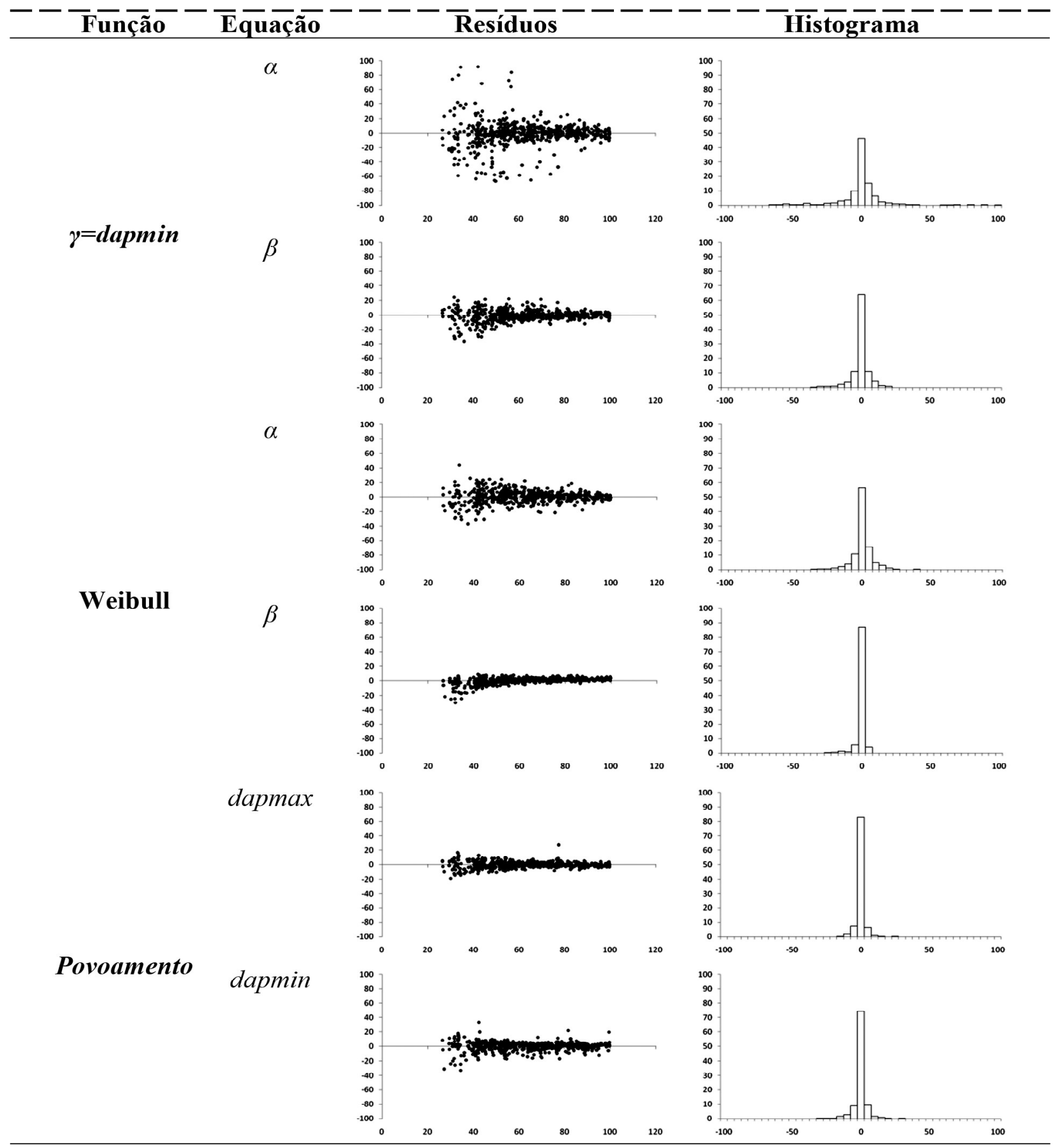

Figura 2 - Dispersão e histograma de resíduos em função da idade do povoamento para as equações que compõem o modelo de distribuição de diâmetros.

Figure 2 -Scatter and histogram of residues according to the age of the stand for the equations that compose the model of distribution of diameters.

Revista Árvore, Viçosa-MG, v.39, n.5, p.943-951, 2015 


\section{REFERÊNCIAS}

BAILEY, R. L.; DELL, T. R. Quantifying diameter distributions with the Weibull function. Forest Science, v.19, n.2, p 97-104,1973.

BETTINGER, P.; BOSTON, K.; SIRY, L.P.; GREBNER, R.L. Forest management and planning. New York: Elsevier, 2009. 331 p.

BINOTI, D.H.B. Funções densidade de probabilidade para a descrição da distribuição diamétrica de povoamentos desbastados de Tectona grandis. 2008. 42f. Monografia (Graduação em Engenharia Florestal) - Universidade Federal de Viçosa, MG, 2008.

CAMPOS, J.C.C.; LEITE, H.G. Mensuração florestal: Perguntas e respostas. $3^{\mathrm{a}}$.ed. Viçosa, MG: Universidade Federal de Viçosa, 2009. 548p.

CAMPOS, J.C.C.; TURNBULL, K. Um sistema para estimar a produção por classe de diâmetro e sua aplicação na interpretação do efeito de desbaste.

Revista Árvore, v.5, n.1, p.1-16, 1981.

CAO, Q.V. Predicting parameters of a Weibull function for modeling diameter distribution. Forest Science, v.50, n.4, p.682-685, 2004.

CLUTTER, J.L.; ALLISON, B.J. A growth and yield model for Pinus radiata in New Zealand for tree and stand simulation. Royal College of Forestry, n.30, p.136-160, 1974.

DAVIS, L.S.; JOHNSON, K.N.; BETTINGER, P.; HOWARD, T.E. Forest management: to sustain ecological, economic, and social values. Illinois: Waveland, 2005. 804p.

GUIMARÃES, D. P. Desenvolvimento de um modelo de distribuição diamétrica de passo invariante para prognose e projeção da estrutura de povoamentos de eucalipto. 1994. 178f. Tese (Doutorado em Ciência Florestal) - Universidade Federal de Viçosa, Viçosa, MG, 1994.

HAFLEY, W.L.; SCHREUDER, H.T. Statistical distributions for fitting diameter and height data in ever-aged stands. Canadian Journal of Forest Research, v.7, p.184-487, 1977.
JOHNSON, N.L.; KOTZ, S.; BALAKRISHNAN, N. Continuous univariate distributions. New York: Applied Probability and Statistics, 1995. $732 \mathrm{p}$.

KNOEBELL, B.R.; BURKHART, H.E.; BECK, D.E.A growth and yield model for thinned stands of yellowpoplar. Forest Science, v.32, n.2, p.62, 1986.

LEITE, H.G.; BINOTI, D.H.B.; GUIMARÃES, D.P.; SILVA, M.L.M.; GARCIA, S.L.R. Avaliação do ajuste das funções Weibull e hiperbólica a dados de povoamentos de eucalipto submetidos a desbaste. Revista Árvore, v.34, n.2, p.305-311, 2010.

MATNEY, T.G.; SULLIVAN, A.D. Variable top volume and height predictions for slash pine trees. Forest Science, v.28, n.2, p.74-82, 1982.

NOGUEIRA, G.S.; LEITE, H.G.; CAMPOS, J.C.C.; CARVALHO, A.F.; SOUZA, A.L. Modelo de distribuição diamétrica para povoamentos de Eucalyptus sp. submetidos a desbaste. Revista Árvore, v.29, n.4, p.579-589, 2005.

PALAHÍ, M.; PUKKALA, T.; BLASCO, E.; TRASOBARES, A. Comparison of beta, Johnson's SB, Weibull and truncated Weibull functions for modeling the diameter distribution of forest stands in Catalonia (north-east of Spain). European Journal of Forest Research, v.126, n.4, p.563-571, 2007.

PALAHÍ, M.; PUKKALA, T.; TRASOBARES, A. Modelling the diameter distribution of Pinus sylvesris, Pinus nigra and Pinus halepensis forest stands in Catalonia using the truncated Weibull function. Forestry, v.79, n.5, p.553-562, 2006.

SHOUKRI, M.M.; MIAN, I.U.M.; TRACY, D.S. Sampling properties of estimators of the log-logistic distribution with application to canadian precipitation data. The Canadian Journal of Statistics, v.16, n.3, p.223-236, 1988.

SIIPILEHTO, J.A. Comparison of two parameter prediction methods for stand structure in Finland. Silva Fennica, v.34, n.4, p.331-349, 2000.

SOKAL, R.R.; ROHLF, F.J. Biometry: the principles and practice of statistics in biological research. 4th ed. New York: W. H. Freeman and, 2012.937p.

Revista Árvore, Viçosa-MG, v.39, n.5, p.943-951, 2015 